\title{
Einfluss von COVID-19 auf die Gruppenprophylaxe in Bayern - eine Querschnittstudie
}

\section{Influence of COVID-19 on group dental prophylaxis activities in Bavaria - a cross-sectional study}

\author{
AUTOREN: NELLY SCHULZ-WEIDNER ${ }^{1}$, MAXIMILIANE AMELIE SCHLENZ², ANNETTE MUSCHLER ${ }^{3}$, BRIGITTE HERMANN ${ }^{3}$, \\ NORBERT KRÄMER' \\ ${ }^{1}$ Universitätsklinikum Gießen und Marburg GmbH, Standort Gießen, Zentrum für Zahn-, Mund- und Kieferheilkunde, \\ Poliklinik für Kinderzahnheilkunde \\ ${ }^{2}$ Universitätsklinikum Gießen und Marburg GmbH, Standort Gießen, Zentrum für Zahn-, Mund- und Kieferheilkunde, \\ Poliklinik für Zahnärztliche Prothetik \\ ${ }^{3}$ Bayerische Landesarbeitsgemeinschaft Zahngesundheit e.V. (LAGZ), Fallstraße 34, 81369 München
}

\section{ZUSAMMENFASSUNG}

Einführung: Die Corona-Virus-Erkrankung (COVID-19) stellt seit mehr als 1 Jahr auch die zahnärztliche Gruppenprophylaxe in Deutschland vor ganz neue Herausforderungen. Durch physische Distanzierungsmaßnahmen konnten die LAGZ-Zahnärztinnen und -ärzte die Kindergärten und Schulen zunächst nicht mehr besuchen, sodass wichtige Impulse für die Zahngesundheit praktisch vollständig entfielen. Es gibt aktuell keine Daten zur Auswirkung der Pandemie auf die Gruppenprophylaxe. Somit war es das Ziel der vorliegenden Studie, die Wahrnehmung von LAGZ-Zahnärztinnen und -ärzten und die Auswirkungen der Pandemie auf ihre gruppenprophylaktischen Tätigkeiten in Bezug auf COVID-19 zu analysieren.

Methode: Die Datenerhebung erfolgte mithilfe eines Online-Fragebogens, in welchem die Perspektive der LAGZ-Zahnärztinnen und -ärzte in Bayern in Bezug auf Prävention, Wohlbefinden und Sicherheit sowie Angst vor Selbst- und Fremdansteckung im Alltag der Gruppenprophylaxe in COVID-19-Zeiten systematisch erfragt wurde.

Ergebnisse: Insgesamt beantworteten 655 Befragte (248 Frauen, 169 Männer, 248 keine Antwort) mit einem durchschnittlichen Alter von 50,2 \pm 10,4 Jahren, einer durchschnittlichen zahnärztlichen Tätigkeit von 23,1 \pm 9,8 Jahren und einer durchschnittlichen gruppenprophylaktischen Tätigkeit von 17,3 \pm 9,5 Jahren den Fragebogen. Dabei führten 9,6 \% der Teilnehmenden gruppenprophylaktische Maßnahmen im Mai 2020 bis Juli 2020 durch. Dieser Anteil blieb ab September 2020 praktisch unverändert (10\%) und steigerte sich ab März 2021 auf 23,3 \%. Während die gruppenprophylaktischen Maßnahmen zunächst nur eingeschränkt v. a. in der Abgabe von Informations- und Zahnputzmaterialien („Reminder-Pakete") erfolgten, änderte sich dies ab Mai 2020, indem auch wieder Besuche der Einrichtungen stattfanden. Ab März 2021 führten die Kolleginnen und Kollegen auch wieder Zahnputzübungen unter Einhaltung der Hygieneregeln durch. In Bezug auf eine mögliche Selbstinfektion gaben 12,6\% der Befragten an, wenigstens 1-mal Sorge gehabt zu haben, sich mit COVID-19 zu infizieren; lediglich 2,4\% erkrankten.

Schlussfolgerung: Die Querschnittstudie zeigt die Schwierigkeiten der LAGZ-Zahnärztinnen und -ärzte in Pandemiezeiten auf und verdeutlicht die Wichtigkeit eines etablierten Konzepts zur dringenden Weiterführung der Gruppenprophylaxe als Pfeiler der Zahnge- sundheit. Somit könnten die gewonnenen Erkenntnisse Basis für die Entwicklung von Konzepten in Pandemiezeiten sein.

\section{Schlüsselwörter: \\ Gruppenprophylaxe, COVID-19, Einschätzung, Selbstinfektion, LAGZ}

\section{ABSTRACT}

Introduction: The 2019 coronavirus disease (COVID-19) has also posed completely new challenges for group dental prophylaxis programs in Germany for more than a year. Due to physical distancing measures, LAGZ (Bavaria Provincial Working Group for Dental Health) dentists were initially no longer able to visit kindergartens and schools, so that important impulses for dental health were practically completely eliminated. There are currently no data on the impact of the pandemic on group prophylaxis. Thus, the aim of the present study was to analyze the perception of LAGZ dentists and the impact of the pandemic on their group prophylactic activities with regard to COVID-19.

Methods: Data collection was carried out with the help of an online questionnaire in which the perspective of the LAGZ dentists in Bavaria 
regarding prevention, well-being, and safety as well as fear of self-infection and infection of others in the daily routine of group prophylaxis during the COVID-19 pandemic were systematically surveyed.

Results: A total of 655 respondents (248 women, 169 men, 248 no response) with an average age of $50.2 \pm 10.4$, an average dental activity of $23.1 \pm 9.8$, and an average group prophylactic activity of $17.3 \pm 9.5$ years answered the questionnaire. In this context, $9.6 \%$ of the participants carried out group prophylactic measures in May 2020 to July 2020. This proportion remained virtually unchanged from September 2020 (10\%) and increased to $23.3 \%$ from March 2021. While group prophylactic measures were initially only limited, mainly in the form of the distribution of information- and toothbrushingmaterials ("reminder packs"), this changed from May 2020 onwards, with visits to kindergartens and schools also taking place again. From March 2021, the colleagues also carried out tooth brushing exercises again in compliance with the hygiene rules. With regard to possible self-infection, $12.6 \%$ of the respondents stated that they had been concerned about becoming infected with COVID-19 at least once; only $2.4 \%$ became ill.

Conclusion: The cross-sectional study shows the difficulties faced by LAGZ dentists during the pandemic and highlights the importance of an established concept for the urgent continuation of group prophylaxis as an important aspect of dental health. Thus, the findings could be the basis for the development of concepts during pandemics.

\section{Keywords:}

Group prophylaxis, COVID-19, Assessment, Self-infection, LAGZ

\section{EINLEITUNG}

Seit nunmehr eineinhalb Jahren stellt die Coronavirus-Krankheit 2019 (COVID-19)-Pandemie, verursacht durch das schwere akute respiratorische Syndrom Coronavirus 2 (SARS-CoV-2), eine Herausforderung für die Gesundheitssysteme auf der ganzen Welt dar [12]. Mehr als $95 \%$ aller Länder weltweit meldeten Infektionen mit dem SARS-CoV-2 [10]. Die erste in Deutschland bestätigte COVID19-Erkrankung wurde im oberbayerischen Landkreis Starnberg am 27.01.2020 verzeichnet und markierte den Beginn der COVID-19-Pandemie in Deutschland [3].

In der Folge beschlossen die meisten Länder physische Distanzierungsmaßnahmen (z. B. Schließung von öffentlichkulturellen sowie Bildungseinrichtungen), um die Infektionsrate zu verlangsamen. In Bayern wurde der Präsenzunterricht an Schulen sogar für 6 Wochen (16.3.2020 bis 26.4.2020) vollständig eingestellt. Darüber hinaus gab es ein Betretungsverbot für Kindertagesstätten [3]. Daher war auch die Gruppenprophylaxe der Bayerischen Landesarbeitsgemeinschaft Zahngesundheit e.V. (LAGZ) unmittelbar von der COVID-19-Pandemie betroffen [2]. Während vor der COVID19-Pandemie LAGZ-Zahnärztinnen und -ärzte regelmäßig Kinder in bayerischen Einrichtungen wie Schulen und Kindergärten im Rahmen der zahnärztlichen Gruppenprophylaxe besuchten, um gruppenprophylaktische Maßnahmen durchzuführen, und darüber hinaus für die Aktion Seelöwe und Löwenzahn als Verweissytem auf den Hauszahnarzt zur Individualprophylaxe motivierten [2], wurde diese Möglichkeit durch die physischen Distanzierungsmaßnahmen stark eingeschränkt. Infolgedessen entfielen diese wichtigen Impulse für die Zahngesundheit von Kindern und Jugendlichen praktisch vollständig. Lediglich LAGZZahnputz- und Unterrichtsmaterialien wurden verteilt, um weiterhin zum Zähneputzen zu motivieren.

Da ein Ende der gegenwärtigen COVID-19-Pandemie in naher Zukunft nicht zu erwarten ist und die Gruppenprophylaxe einen wichtigen Anteil zur Kariesprävention im Kindes- und Jugendalter beiträgt, wird vonseiten der LAGZ - trotz Pandemie - die Weiterführung der Gruppenprophylaxe unter zusätzlichen Hygiene- und Schutzmaßnahmen dringend empfohlen. In Anlehnung an die Richtlinien zur Infektionskontrolle des Deutschen Arbeitskreises für Hygiene in der Zahnmedizin im bereits etablierten Hygienekonzept der Zahnheilkunde [5], welches sich auch bei verschie- denen anderen Infektionskrankheiten (z. B. Tuberkulose) bewährt hat, wurden von der LAGZ folgende Empfehlungen ausgesprochen:

- Das tägliche Zähneputzen in den Einrichtungen wurde auch im Hinblick auf die Rolle der Mundgesundheit bei der Abwehr im Infektionsgeschehen angeraten.

- Unter Berücksichtigung der Empfehlung der Deutschen Arbeitsgemeinschaft für Jugendzahnpflege e.V. (DAJ) "Mundhygiene in Zeiten von COVID-19 - jetzt erst recht!“ [8] wurden über die sog. „Handreichung für LAGZ-Zahnärzte" Hinweise für das Beibehalten bzw. Neueinführen des Zähneputzens in den Einrichtungen ausgesprochen.

- Es wurde ein sog. „Reminder-Paket“ entwickelt, welche die Kontaktpflege per E-Mail oder Telefon mit den Einrichtungen zur Abfrage bezüglich Materialbedarf für die ab Mai 2020 in den Einrichtungen befindlichen Kinder steuerte. Die anschließende Übergabe der Zahnbürsten und Zahnpasten in der Einrichtung sollte hierbei den Einrichtungskontakt unterstützen.

Da über einen langen Zeitraum die Infektionslage nicht klar abzuschätzen war, erfolgten bis Juni 2021 keine Praxisbesuche der in zahnärztlicher Gruppenprophylaxe betreuten Kinder, jedoch wurde der Zahnarztbesuch im Rahmen der Individualprophylaxe Kindern und Eltern ausdrücklich empfohlen (s. auch Aktion Seelöwe und Löwenzahn) [2].

Ab dem 07.06.2021 konnten bei entsprechenden Inzidenzzahlen alle bayerischen Schülerinnen und Schüler in den Präsenzunterricht zurückkehren, sodass in Absprache mit den Leitungen unter Berücksichtigung aller Hygienemaßnahmen eine Ausübung der Gruppenprophylaxe inklusive Praxisbesuch wieder möglich war. Die „Reminder-Pakete“ als Kontaktmöglichkeit und Motivationstool waren bei Nicht-Realisierbarkeit der Maßnahmen noch bis Ende Juli 2021 erhältlich.

Nach bestem Wissen der Autoren gibt es keine systematische Untersuchung zur Gruppenprophylaxe in der COVID19-Pandemie. Daher war es das Ziel dieser Querschnittstudie, alle LAGZ-Zahn- 
ärztinnen und -ärzte hinsichtlich der Aspekte Betreuung der Einrichtungen, Sicherheitsmaßnahmen, Angst vor Selbst- bzw. Fremdansteckung sowie Perspektiven zur zukünftigen Durchführung zu befragen und die Ergebnisse systematisch für alle Kolleginnen und Kollegen aufzubereiten. Hieraus können sich Hinweise ergeben, wie die Gruppenprophylaxe trotz COVID-19-Pandemie weitergeführt werden kann, um die Zahngesundheit von Kindern und Jugendlichen auch in Pandemiezeiten weiter zu unterstützen.

\section{MATERIAL UND METHODE}

Für die Online-Umfrage wurden 2438 LAGZ-Zahnärztinnen und -ärzte aus 92 Arbeitskreisen im Bundesland Bayern befragt, die im Rahmen der Gruppenprophylaxe tätig sind.

Es wurde eine fragebogenbasierte Umfrage verwendet, die in Zusammenarbeit mit der Servicestelle Lehrevaluation der Justus-Liebig-Universität Gießen entwickelt und über ein Online-Umfragetool (LimeSurvey, Hamburg, Deutschland) bereitgestellt wurde. Der generierte Link wurde von der Geschäftsstelle der LAGZ per E-Mail zunächst an alle $92 \mathrm{Ar}$ beitskreisvorsitzenden weitergeleitet mit der Bitte, alle LAGZ-Zahnärztinnen und -ärzte zur Teilnahme an dieser Umfrage einzuladen.

Die retrospektive Umfrage umfasste Multiple-Choice-Fragen zu COVID-19 und zahnmedizinischer Prävention einschließlich zusätzlicher Sicherheitsmaßnahmen, Wohlbefinden und Sicherheit sowie Angst vor Selbst- und Fremdansteckung und abschließende demografische Fragen. Für die systematische Auswertung wurde eine 5-stufige Likert-Skala [9] verwendet, wobei eine Enthaltung möglich war. Die erhobenen Daten waren vollständig anonym, sodass zu keinem Zeitpunkt ein Rückschluss auf die Teilnehmenden möglich war. Am 23.06.2021 wurde die Umfrage gestartet, wobei nach 14 Tagen eine Erinnerung an alle Teilnehmenden verschickt wurde. Die Umfrage wurde am 31.07.2021 abgeschlossen, und nur vollständig ausgefüllte Fragebögen wurden für die Datenauswertung berücksichtigt.

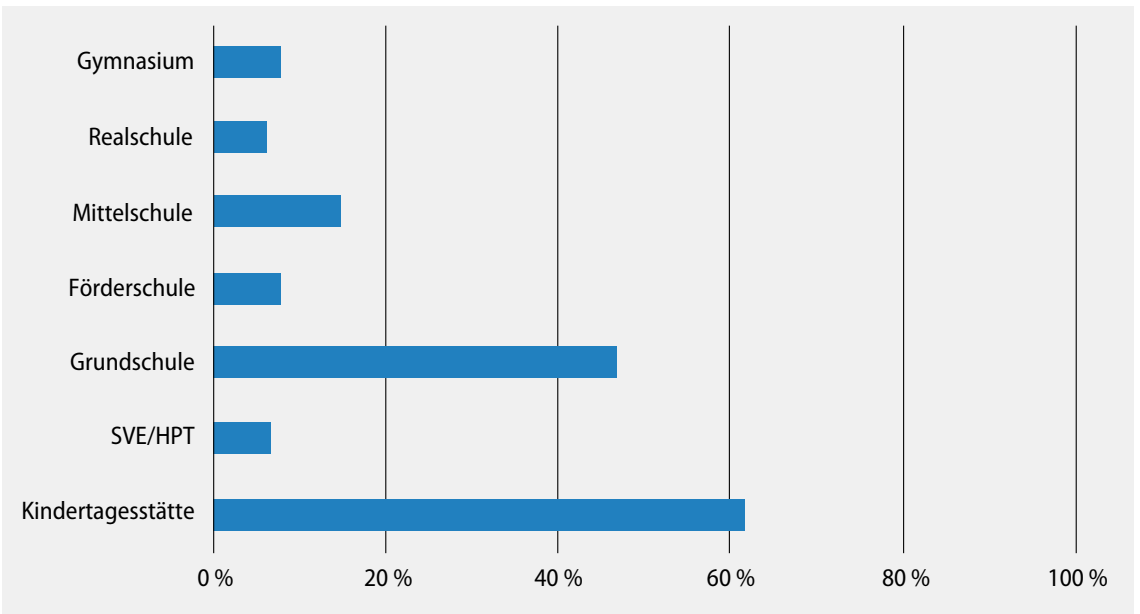

Abbildung 1 Verteilung der betreuten Einrichtungen der LAGZ-Zahnärztinnen und -ärzte in Prozent vor der COVID-19-Pandemie (Mehrfachantworten möglich) SVE/HTP Schulvorbereitende Einrichtung/ Heilpädagogische Tagesstätte

Figure 1 Percentages and types of child care centers served by LAGZ dentists prior to the COVID-19 pandemic (multiple responses possible). SVE/HPT elementary school preparation classes

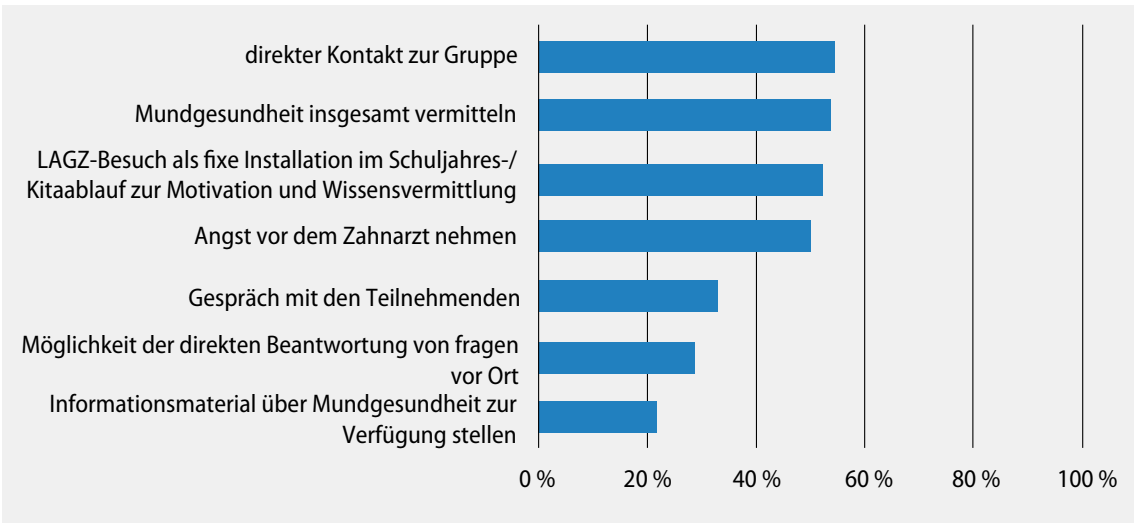

Abbildung 2 Aspekte der Gruppenprophylaxe nach Wichtigkeit geordnet (Mehrfachantworten möglich) Figure 2 Aspects of group prophylaxis ranked by importance (multiple responses possible)

Zur Zusammenfassung und Analyse der Daten wurden deskriptive Statistiken verwendet. Außerdem wurden die Daten in Bezug auf die Angst vor Selbst- und Fremdansteckung weiter analysiert. Absolute und relative Häufigkeiten sowie Maße der zentralen Tendenz und der Streuung wurden entsprechend dem Skalenniveau und der Verteilung der einzelnen Variablen berechnet.

\section{ERGEBNISSE}

Soziodemografische Daten und berufsbezogene Merkmale

Von 2438 LAGZ-Zahnärztinnen und -ärzten, die zur Teilnahme eingeladen wurden, haben $26,9 \%(n=655)$ geantwortet. Davon waren 248 weiblich, 169 männlich, und 248 gaben keine Antwort auf die Frage. Das Durchschnittsalter der Teilnehmenden betrug 50,2 $\pm 10,4$ Jahre. Dabei reichte die Anzahl an Jahren in der zahnärztlichen Praxis von 1 bis 50 Jahre $(23,1 \pm$ SD 9,8), und die Dauer der gruppenprophylaktischen Tätigkeit betrug $17,3 \pm 9,5$ Jahre.

Insgesamt zählten sich 17 \% der Kolleginnen und Kollegen zu einer Risikogruppe [7] in Bezug auf eine COVID19-Infektion. Diese begründeten ihre Einschätzung aufgrund von chronischen Krankheiten ( $\mathrm{n}=38)$, Alter $(\mathrm{n}=80)$, Fettleibigkeit $(n=15)$, Rauchen $(n=4)$, Im- 


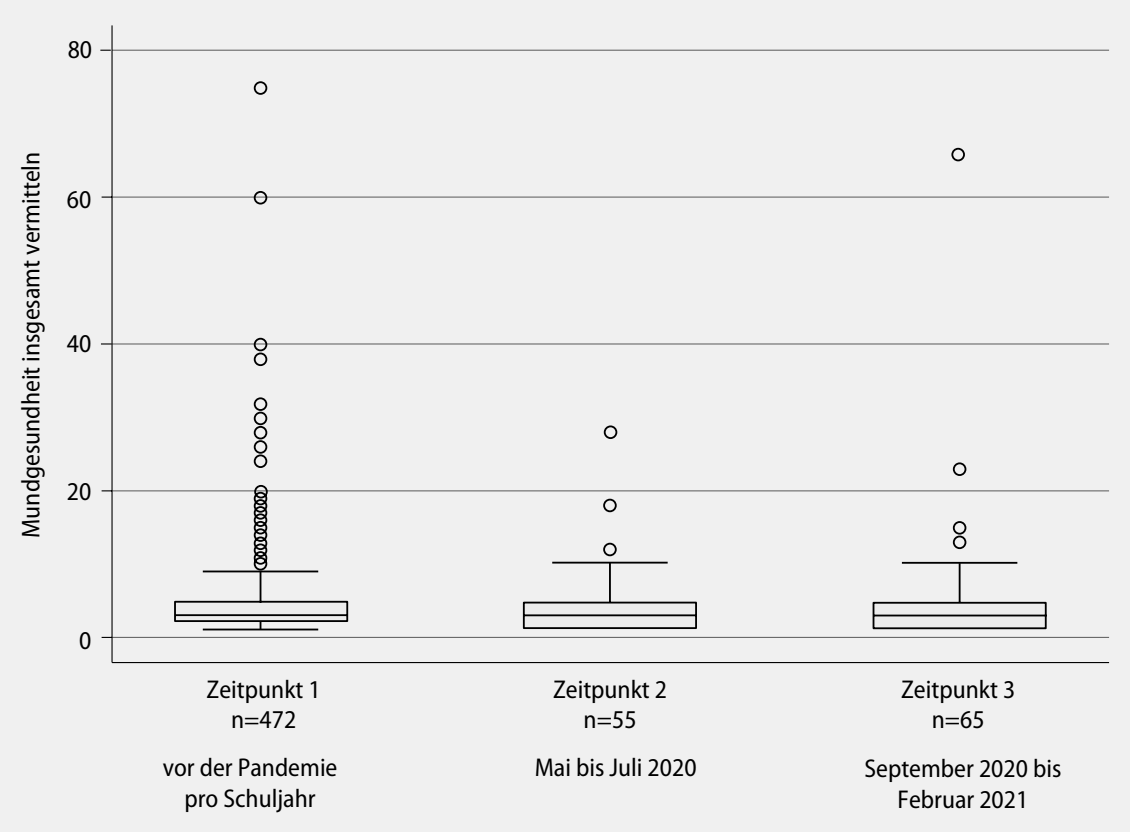

Abbildung 3 Angegebene Einrichtungsbetreuungen der LAGZ-Zahnärztinnen und -ärzte im Verlauf der COVID-19-Pandemie ( $n=$ Anzahl der abgegebenen Antworten)

Figure 3 Number of schools visited by LAGZ dentists during the COVID-19 pandemic ( $n=$ number of responses given)

munsuppression $(\mathrm{n}=3)$ oder Schwangerschaft $(n=3)$. Als weitere Gründe für die Einstufung in eine Risikogruppe wurden der eigene Patientenkontakt mit Aerosolbehandlung, die Behandlung von evtl. asymptomatischen Kindern bei COVID19-Infektion sowie eine eigene Tumorerkrankung genannt.

\section{Informationsmedien in Bezug auf COVID-19}

Die LAGZ-Zahnärztinnen und -ärzte informierten sich v. a. über das Robert Koch-Institut (RKI) (57,1\%), verschiedene zahnärztliche Verbände (46,5\%), Tageszeitungen oder Zeitschriften (33,5\%), Fachliteratur (33,1 \%) sowie kollegialen Austausch (27,7\%). Auch soziale Medien und Podcasts gaben über $29 \%$ der Teilnehmenden als Informationsmedium an.

\section{Gruppenprophylaktische Maßnahmen vor der Pandemie}

Insgesamt betreuten die LAGZ-Zahnärztinnen und -ärzte vor der Pandemie 3 (Median; Interquartilbereich 2 bis 5) Einrichtungen/Klassen/Gruppen pro Schuljahr (s. auch Abb. 3). Dabei war v. a. die Kindertagesstätte die meistbetreute Ein- richtung, gefolgt von den Grundschulen (Abb. 1).

Im Hinblick auf die Vermittlung von Zahngesundheit gaben die LAGZ-Zahnärztinnen und -ärzte an, dass v. a. der direkte Kontakt mit der Gruppe (54,4\%), die Vermittlung von Mundgesundheit insgesamt (53,8 \%), der LAGZ-Besuch als fixe Installation im Schuljahres-/Kitaablauf zur Motivation und Wissensvermittlung (52,3 \%) sowie der Aspekt, dem Kind die Angst vor dem Zahnarzt zu nehmen (50,2 \%), als wichtigste Pfeiler der Gruppenprophylaxe angesehen wurden (Abb. 2).

\section{Einfluss von COVID-19 auf die Maßnahmen}

Nach den ersten Einrichtungsschließungen gaben 9,6 \% der Teilnehmenden für den Zeitraum Mai 2020 bis Juli 2020 an, gruppenprophylaktische Maßnahmen zumindest mit Einschränkungen durchgeführt zu haben. Von diesen machte die Mehrzahl v. a. von der Materialbestellung online oder per Formular mit Übergabe des „Reminderpakets“ in der Einrichtung Gebrauch (81,4 \%). Diejenigen, die angaben, Einrichtungen im ersten Zeitraum betreut $z u$ haben $(n=55)$, taten dies im
Median von 3 (Median Wert 3; Interquartilbereich 1-5) Maßnahmen/Gruppenbetreuungen/Klassenbetreuungen von Mai 2020 bis Juli 2020. Nach den Sommerferien 2020 blieb der Anteil der durchgeführten gruppenprophylaktischen Maßnahmen praktisch unverändert (10 \% mit einer Anzahl von 3 [Median Wert 3; Interquartilbereich 1-5] Maßnahmen/Gruppenbetreuungen/Klassenbetreuungen von September 2020 bis Februar 2021) (Abb. 3). Dabei wurde in der Mehrheit der Besuch der Einrichtungen unter Einhaltung der Hygieneregeln ohne Zahnputzübungen (67,1\%), gefolgt von der Motivation zur Teilnahme an der Aktion Seelöwe und Löwenzahn (47,1 \%) durchgeführt. Ab März 2021 zeigte sich in $23,2 \%$ eine gruppenprophylaktische Betreuung unter den Kolleginnen und Kollegen der LAGZ, und zwar hiervon in $68,2 \%$ als Übergabe des „Reminderpakets“, gefolgt vom Besuch der Einrichtungen unter Einhaltung der Hygieneregeln mit Zahnputzübungen $(63,6 \%)$ sowie der Motivation zur Teilnahme an der Aktion Seelöwe und Löwenzahn (49,4 \%).

Es gaben 27,3\% der LAGZ-Zahnärztinnen und -ärzte an, dass seit März 2020 bis Juli 2021 das Zähneputzen auf Einrichtungsseite aufgrund der Hygieneauflagen nicht durchgeführt wurde. Die Abb. 4 zeigt die Einschätzung der Umfrageteilnehmer in Bezug auf die Frage, in welcher Form dieser wichtige Impuls wieder eingeführt werden könnte.

Es gaben 54,8\% der Teilnehmenden an, dass gruppenprophylaktische Maßnahmen aufgrund von COVID-19 verschoben wurden. Von diesen verschobenen Terminen wurden 53,6 \% von Einrichtungsseite aus abgesagt, in 13,4 \% von den LAGZZahnärztinnen und -ärzten, in beiden Fällen aus Sorge vor einer möglichen Infektion von Kindern und Erwachsenen. In 23,5\% waren die zu betreuenden Einrichtungen sogar geschlossen.

\section{COVID-19-Infektion}

Im Hinblick auf eine mögliche Selbstinfektion gaben 12,6\% der Befragten an, mindestens 1-mal Angst vor einer Ansteckung mit COVID-19 in einer Einrichtung gehabt zu haben; nur 2,4\% der LAGZ-Zahnärztinnen und -ärzte er- 
krankten an COVID-19. Dabei gaben 8 Personen an, sich im privaten Umfeld angesteckt zu haben und 5 in der eigenen zahnärztlichen Praxis.

Mithilfe unseres Fragebogens haben wir zudem im Zeitraum März 2020 bis Juni 2021 die Angst, sich selber in Einrichtungen zu infizieren, (gemessen nach der Likert-Skala) retrospektiv analysiert. Es zeigte sich ein deutlicher Anstieg der Angst zum Zeitpunkt höherer Infektionszahlen in Bayern (Abb. 5).

In Bezug auf die Sorge vor Selbstinfektion gaben $34,7 \%$ der befragten LAGZZahnärztinnen und -ärzte an, erst dann wieder mit Maßnahmen starten zu wollen, wenn ein eigener vollständiger Impfschutz vorläge. Dabei waren bereits 57,4 \% aller Teilnehmenden doppelt geimpft.

Zusätzlich gaben $66 \%$ der befragten LAGZ-Zahnärztinnen und -ärzte an, dass sie bei Wiederaufnahme der regelhaften Gruppenprophylaxe zunächst Mundhygieneinstruktionen (66\%), gefolgt von Ernährungslenkungen (55,6 \%), Besuch der Praxis $(23,5 \%)$ und Fluoridierung (15\%) schwerpunktmäßig durchführen würden.

\section{Digitalisierung}

Es hatten $49 \%$ der Kolleginnen und Kollegen bereits Erfahrung mit digitalen Formaten in Form von Meetings/Konferenzen gesammelt. Darüber hinaus konnten sich $17,7 \%$ vorstellen, auch gruppenprophylaktische Impulse über digitale Formate durchzuführen. Vorstellbare Impulse waren dabei die theoretische Unterrichtsgestaltung (z. B. Ernährungsberatungen), gefolgt von Informationsnachmittagen und/oder -abenden sowie die praktische Unterrichtsgestaltung in Form von Zahnputzübungen.

\section{Zusätzliche Infektionsschutzmaßnahmen}

Als genutzte Infektionsschutzmaßnahmen gab die Mehrheit eine zusätzliche Händehygiene und das Tragen einer FFP2-Maske in der Einrichtung an. Der Corona-Abstrich vor der Maßnahme und das Tragen eines Visiers/Schutzschildes wurden dahingegen in der Mehrzahl als nicht sinnvoll erachtet (Abb. 6).

Darüber hinaus zeigte sich, dass die zusätzlichen Schutzmaßnahmen die Mi-

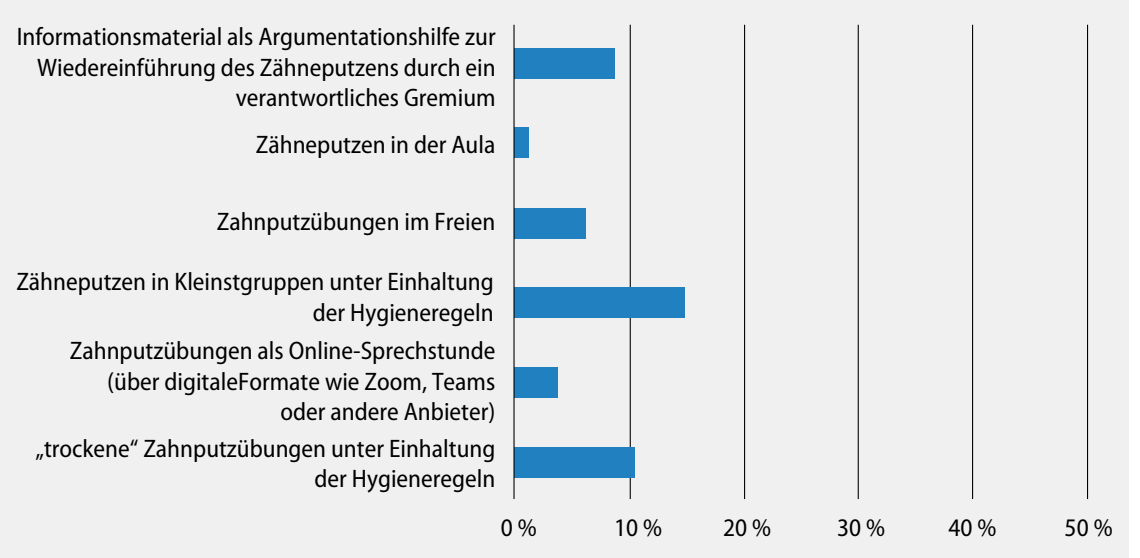

Abbildung 4 Relative Häufigkeit der Wiedereinführungsmöglichkeiten des Zähneputzens Figure 4 Relative frequencies of newly introduced options for tooth-brushing exercises mik und die Kommunikation beeinträchtigten. Die Kolleginnen und Kollegen fühlten sich bei der Durchführung der Gruppenprophylaxe im Rahmen der COVID-19-Pandemie unwohl, ebenso wie sie einschätzten, dass sich das Team der Einrichtungen unwohl fühlte.

Nur 16,5 \% der Befragten hielten es für gut, die Gruppenprophylaxe unter
Pandemiebedingungen beizubehalten (Abb. 7).

Unabhängig von den oben genannten Aspekten gab die Mehrheit der Befragten an, dass sie nach der COVID-19-Pandemie je nach Situation zusätzliche Schutzmaßnahmen in Bezug auf andere durch die Luft übertragene Infektionskrankheiten (z. B. Tuberkulose) beibehalten würden.
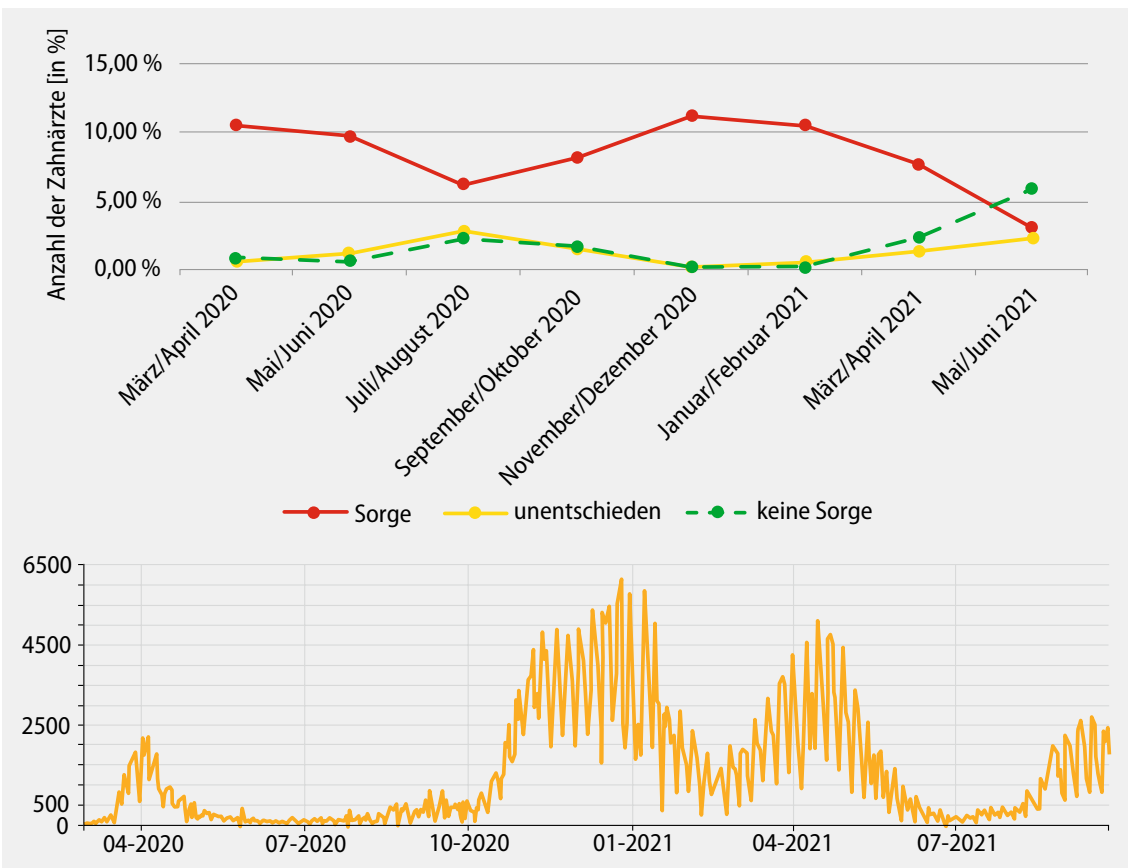

Abbildung 5 Sorge vor Selbstinfektion in der Einrichtung (a) im Verhältnis zu den Inzidenzzahlen in Bayern nach Daten des RKI (b) [14]

Figure 5 Concern about becoming infected in a child care center (a) vs. incidence in Bavaria based on data from the Robert Koch-Institut (b) [14] 


\section{DISKUSSION}

Es gibt erste Hinweise, dass die Häufigkeit von Milchzahnkaries in COVID19-Zeiten zugenommen hat [13]. Zur Prävention der ECC spielt die traditionelle Gesundheitserziehung mit Instruktionen sowohl der Kinder als auch der Erzieherinnen und Erzieher vor Ort eine entscheidende Rolle [15]. Die positive Wirkung von Programmen auf die Mundgesundheit der Kinder und der ökonomische Effekt wurden dabei bereits in Studien aus Brasilien und Chile belegt [4, 11]. Darüber hinaus belegten Antonio et al. den nachteiligen Effekt einer 24-monatigen Unterbrechung eines bestehenden Prophylaxeprogrammes. Dabei zeigte sich, dass selbst 12 Monate nach der Unterbrechung die ursprünglich gemessenen niedrigen Gingivitisparameter nicht erreicht werden konnten [1]. Dies



Abbildung 6 Zusätzliche Maßnahmen zum Infektionsschutz

Figure 6 Additional measures taken to protect against infection

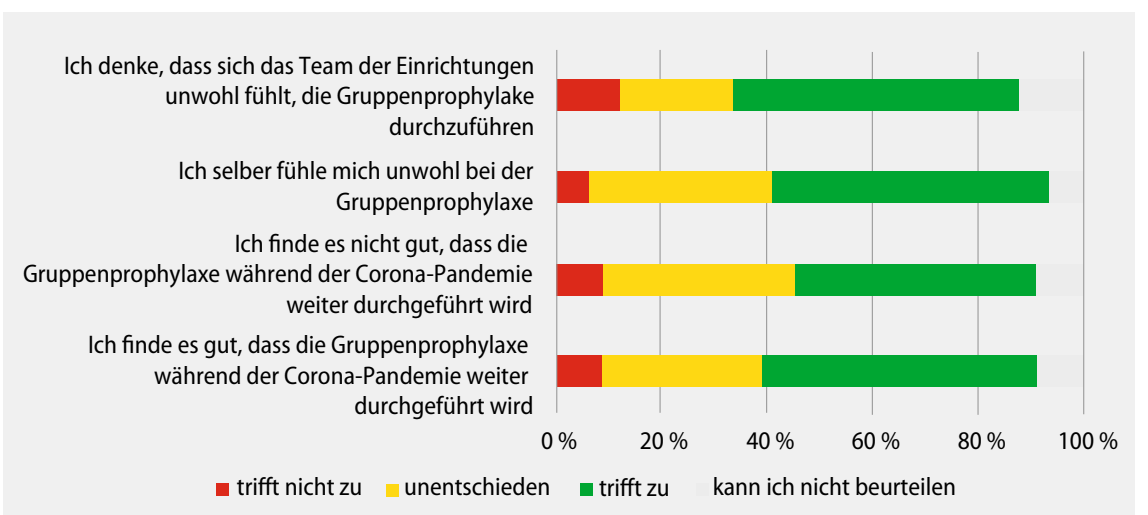

Abbildung 7 Perspektive der Teilnehmer auf die Weiterführung der Gruppenprophylaxe in Pandemiezeiten Figure 7 Opinions of participants on the continuation of group prophylaxis activities during the pandemic weshalb die Perspektive nicht direkt übertragen werden kann.

In Anbetracht der Tatsache, dass $12,6 \%$ der Kollegen mindestens 1-mal angaben, sich über eine Selbstinfektion mit COVID-19 in der Einrichtung Sorgen gemacht zu haben, scheint dies ein ernstes Problem zu sein und unterstreicht die Bedeutung einer ausreichenden Infektionskontrolle. Entsprechend der Anzahl der bestätigten COVID-19-Fälle in Bayern konnten wir zudem ein höheres Maß an Angst vor Selbstansteckung in den Monaten April 2020 sowie im November und Dezember 2020 zeigen.

Die fehlende Impfmöglichkeit verbunden mit der Schwierigkeit der Testung von Kleinkindern aufgrund mangelnder Kooperation und den möglichen asymptomatischen COVID-19-infizierten Kindern zeigt [16], dass gerade die mit der Gruppenprophylaxe betreuten Kolleginnen und Kollegen mit der Herausforderung eines erhöhten Selbstinfektionsrisikos umgehen müssen. Der eigene Impfstatus spielte hier in der Frage der Infektionssorge bzw. Weiterführung der gruppenprophylaktischen Tätigkeit eine große Rolle. So gaben $34,7 \%$ an, die gruppenprophylaktische Tätigkeit dann wieder aufnehmen zu wollen, wenn sie vollständig geimpft seien. Im Hinblick auf den Impfstatus zeigt die Studie den im Vergleich zur allgemeinen Bevölkerung in Bayern höheren vollständigen Impfschutz mit 57,7 \% im Juli 2021 [6].

COVID-19 erfordert in der Gruppenprophylaxe ebenso wie in der zahnärztlichen Praxis ein angepasstes Konzept zur Weiterführung und Sicherstellung der wichtigen Präventionspfeiler. Die Tatsache, dass in über $27 \%$ das Zähneputzen in den Einrichtungen aufgegeben wurde, zeigt auch hier die Sorge einer möglichen Infektion und unterstreicht die Notwendigkeit von Fachkompetenz und Ideen, um diesen wichtigen Impuls der Gruppenprophylaxe aufrechtzuerhalten. Unter Berücksichtigung der zusätzlichen Herausforderung für die Kolleginnen und Kollegen zeigt sich in Pandemiezeiten die Bereitschaft, sich auf neue Konzepte einzulassen, um die Zahn- und Mundgesundheit zu unterstützen. So wurde das sog. „Reminderpaket“ als Kontaktpflege- 
tool gut angenommen, und darüber hinaus wurden neue konzeptionelle Ideen für das Zähneputzen oder andere Informationseinheiten durch digitale Impulse oder andere Aspekte ins Auge gefasst.

Die zusätzlichen Schutzmaßnahmen wie verbesserte Händehygiene und FFP2-Masken, die als sehr nützlich für die Infektionskontrolle angesehen wurden, waren andererseits eine Belastung, insbesondere als eingeschränkter Kommunikationsaspekt durch das Fehlen der Mimik. Trotzdem besteht ein breiter Konsens darüber, dass diese Schutzmaßnahmen auch nach dem Abklingen der COVID-19-Pandemie weitergeführt werden sollten.

Da alle Teilnehmer darüber informiert wurden, dass die Datenerhebung völlig anonym war und keine Rückverfolgung zuließ, wurde eine Antwortverzerrung verhindert. Eine Einschränkung dieser Studie besteht darin, dass die Datenerhebung innerhalb eines Monats abgeschlossen wurde. Insbesondere die retrospektive Frage nach der Angst vor einer Ansteckung mit COVID-19 stellt hiermit das Risiko einer verzerrten retrospektiven Darstellung dar. Bei der Interpretation der Ergebnisse sollte somit berücksichtigt werden, dass es sich um eine retrospektive Befragung handelt. Die Fragen wurden anhand einer Likert-Skala ausgewertet, die ein Standardverfahren für Umfragen im medizinischen Bereich ist [9].

Als Ausblick gab die Mehrzahl der Befragten an, dass sie Mundhygieneinstruktionen als Schwerpunkt in der Gruppenprophylaxe durchführen würden und planen, hiermit regelhaft als erstem Impuls zu starten. Zusätzlich unterstreicht die positive Antwort der Mehrheit aller Umfrageteilnehmer zu der Frage, ob sie sich vor einer Ansteckung mit COVID-19 durch zusätzliche Schutzmaßnahmen geschützt fühlen, den hohen Standard der bereits etablierten und vertrauten zusätzlichen Schutzmaßnahmen. Der zusätzliche Schutz durch Impfung in der Kollegenschaft scheint darüber hinaus ein wichtiger Aspekt in der Betreuung von Kindern in der Gruppenprophylaxe zu sein und gibt, bedingt durch die im Verhältnis zur Bevölkerung hohe Impfquote, eine positive Prognose in Bezug auf die wichtige Wiederaufnahme von gruppenprophylaktischen Maßnahmen.

\section{SCHLUSSFOLGERUNG}

Diese Querschnittstudie gibt einen Überblick über die Schwierigkeiten der Durchführung der Gruppenprophylaxe aus der Sicht der betreuenden Zahnärztinnen und Zahnärzte. Die dringende Weiterführung der Gruppenprophylaxe mit Gesundheitserziehung in den Einrichtungen vor Ort sollte sich daher an die aktuellen Hygieneanforderungen anlehnen, die auch in den zahnärztlichen Praxen umgesetzt werden, um einer möglichen Infektion durch Kinder vorzubeugen.

\section{KORRESPONDENZADRESSE}

Dr. Nelly Schulz-Weidner

Universitätsklinikum Gießen und Marburg GmbH, Standort Gießen

Zentrum für Zahn-, Mund- und Kieferheilkunde Poliklinik für Kinderzahnheilkunde

Schlangenzahl 14, 35392 Gießen

Nelly.Schulz-Weidner@dentist.med.uni-giessen.de

ORCID ID 0000-0003-4562-8232

\section{Interessenkonflikt}

Die Autoren geben an, dass kein Interessenkonflikt besteht.

\section{Datenverfügbarkeit}

Alle im Rahmen dieser Studie gewonnenen oder analysierten Daten sind in diesem veröffentlichten Artikel enthalten.

\section{LITERATUR}

1. Antonio, AG, Kelly, A, Valle, DD, Vianna, RB, Quintanilha, LE. Long-term effect of an oral health promotion program for schoolchildren after the interruption of educational activities. J Clin Pediatr Dent 2007; 32:37-41

2. Bayerische Arbeitsgemeinschaft Zahngesundheit e.V. 2021; Available from: http://www.lagz.de; Zugriff am 01.10.2021

3. COVID-19-Pandemie in Bayern. Wikepedia.org.; Available from: https://de.wikipedia.org/wiki/ COVID-19-Pandemie_in_Bayern.ZZugriff am 01.10 .2021

4. de Farias, IA, de Araujo Souza, GC, Ferreira, MA. A health education program for Brazilian public schoolchildren: the effects on dental health practice and oral health awareness. J Public Health Dent 2009; 69:225-230

5. Deutscher Arbeitskreis für Hygiene in der Zahnmedizin (Hrsg.) Hygieneleitfaden, 14. Ausgabe 2021. Available from: https://dahz.org/ hygieneleitfaden. Letzter Zugriff am 02.10.2021

6. Epidemiologischer SARS-CoV-2 Situationsbericht. 2021. Lebensmittelssicherheit, B.L.f.G.u., Available from: https://www.lgl.bayern.de. Letzter Zugriff am 02.10.2021
7. European Centre for Disease Prevention and Control. High-Risk groups for COVID-19. Available from: www.ecdec.europa.eu/en/covid-19/highrisk-groups. Letzter Zugriff am 01.10.2021

8. Institut für Hygiene und Öffentliche Gesundheit der Universitätsklinik Bonn, BZÖG, DAJ (Hrsg.) Mundhygiene in Zeiten von COVID-19 - jetzt erst recht! Available from: https://www.daj.de/ fileadmin/user_upload/PDF_

Downloads/2021_08_25_Zähnpflege_ Gemeinschaftseinrichtungen_Druck.pdf. Letzter Zugriff am 06.10.2021

9. Komorita, S. Attitude content, intensity, and the neutral point on a likert scale. J Soc Psychol 1963; (61):327-334

10. Li Q, Guan X, Wu P, Wang X, Zhou L, Tong Y, Ren R, Leung KSM, Lau EHY, Wong JY, Xing $X$, Xiang $N$, Wu Y, Li C, Chen Q, Li D, Liu T, Zhao J, Liu M, Tu W, Chen C, Jin L, Yang R, Wang Q, Zhou S, Wang R, Liu H, Luo Y, Liu Y, Shao G, Li H, Tao Z, Yang Y, Deng Z, Liu B, Ma Z, Zhang Y, Shi G, Lam TTY, Wu JT, Gao GF, Cowling BJ, Yang B, Leung GM, Feng Z. Early Transmission Dynamics in Wuhan, China, of Novel Coronavirus-Infected Pneumonia. N Engl J Med 2020 Mar 26;382(13):1199-1207. https://doi.org/10.1056

11. Marino, R, Fajardo, J, Morgan, M. Costeffectiveness models for dental caries prevention programmes among Chilean schoolchildren. Community Dent Health 2012;29:302-308

12. Meng L, Hua F, Bian Z. Coronavirus Disease 2019 (COVID-19): Emerging and Future Challenges for Dental and Oral Medicine. J Dent Res 2020; 99(5):481-487. doi: 10.1177/0022034520914246

13. Ren YF, Rasubala L, Malmstrom H, Eliav E. Dental Care and Oral Health under the Clouds of COVID19. JDR Clin Trans Res. 2020; 5(3):202-210. https:// doi.org/110.1177/2380084420924385

14. RKI. COVID-19: Fallzahlen in Deutschland und weltweit. https://www.rki.de/DE/Content/ InfAZ/N/Neuartiges_Coronavirus/Fallzahlen. html. Letzter Zugriff 06.10.2021

15. Stein, C, Santos, NML, Hilgert, JB, Hugo, FN. Effectiveness of oral health education on oral hygiene and dental caries in schoolchildren: Systematic review and meta-analysis. Community Dent Oral Epidemiol 2018;46:30-37

16. Zimmermann P, Curtis, N. Coronavirus Infections in Children Including COVID-19: An Overview of the Epidemiology, Clinical Features, Diagnosis, Treatment and Prevention Options in Children. Pediatr Infect Dis 2020; 39(5):355-368 
Hier steht eine Anzeige.

算 Springer 